\author{
S. E. R. Mons. Rino Fisichella
}

\title{
QUESTO È IL TEMPO DELLA MISERICORDIA
}

Il richiamo al tema della misericordia ha trovato senza dubbio un grande eco in questo Giubileo straordinario; eppure, è utile non dimenticare che è stato preparato negli ultimi decenni soprattutto con il forte impulso dato da s. Giovanni Paolo II. Per creare lo scenario più coerente a questo "tempo della misericordia" su cui vogliamo riflettere è necessario ritornare all'enciclica Dives in misericordia. Due espressioni di quel testo, in modo particolare, possono aiutare a identificare la nostra problematica.

La prima dice:

La mentalità contemporanea, forse più di quella dell'uomo del passato, sembra opporsi al Dio di misericordia e tende altresì ad emarginare dalla vita e a distogliere dal cuore umano l'idea stessa della misericordia. La parola e il concetto di misericordia sembrano porre a disagio l'uomo, il quale, grazie allenorme sviluppo della scienza e della tecnica [...] è diventato padrone ed ha soggiogato e dominato la terra. Tale dominio sulla terra, inteso talvolta unilateralmente e superficialmente, sembra che non lasci spazio alla misericordia ${ }^{1}$.

Una considerazione profondamente vera se si considera che la misericordia non trova riscontro nei dizionari di teologia e sembra relegata a un'appendice degli attributi di Dio o ad un breve capitolo della spiritualità. La teologia ha dimenticato per molto tempo la misericordia e ne è scaturita una oggettiva povertà nella presentazione della fede.

Una seconda espressione si trova a conclusione dell'enciclica dove il santo $\mathrm{Pa}$ pa afferma:

1 Dives in misericordia, 2 [DM], http://w2.vatican.va/content/john-paul-ii/it/encyclicals/ documents/hf_jp-ii_enc_30111980_dives-in-misericordia.html (accesso il 03.08.2017). 
E se taluno dei contemporanei non condivide la fede e la speranza che mi inducono, quale servo di Cristo e ministro dei misteri di Dio, a implorare in questa ora della storia la misericordia di Dio per l'umanità, egli cerchi almeno di comprendere il motivo di questa premura. Essa è dettata dall'amore verso l'uomo, verso tutto ciò che è umano e che, secondo l'intuizione di gran parte dei contemporanei, è minacciato da un pericolo immenso ${ }^{2}$.

Per alcuni versi, Giovanni Paolo II prendeva la misericordia come condizione di condivisione con gli uomini del nostro tempo per far comprendere l'anelito della Chiesa alla salvaguardia del creato e dell'uomo in esso. La misericordia quindi come esperienza comune agli uomini, che può permettere un dialogo e un impegno comune per il futuro dell'umanità.

Con ragione, pertanto, si può parlare di una preparazione nella vita della Chiesa a percepire la ricchezza che proviene dalla misericordia e pensare questo tempo come quello della misericordia. Non si può sottacere, infine, il valore simbolico di alcuni gesti. Nel grande Giubileo del 2000 il segno più eloquente fu certamente la canonizzazione di Suor Faustina, mentre in questo Giubileo straordinario quella di Madre Teresa. L'uno e l'altro esempio, comunque, non sono altro che espressione della misericordia. Suor Faustina rappresenta la mistica che ha fatto scoprire i tesori della misericordia divina; Madre Teresa, quella che ha fatto della sua vita un segno concreto della misericordia. La continuità di questa icona permette di riflettere su come far diventare il tempo della Chiesa un tempo di misericordia.

\section{Il tempo della misericordia}

"Ecco perché il Giubileo: perché questo è il tempo della misericordia" ${ }^{3}$. L'espressione nella sua semplicità sintetizza il pensiero di Papa Francesco. È necessario entrare nel merito di questo orizzonte per cercare di vedere in quale modo la misericordia può diventare la cifra su cui leggere, interpretare e vivere l'esperienza cristiana. Sappiamo che la misericordia non si lascia rinchiudere nell'unico termine che la designa. Anzi, nel momento stesso in cui volessimo definire la misericordia - darle cioè una descrizione che ne esaurisce il contenuto - l'avremmo definitivamente distrutta. La misericordia ha bisogno di rimanere aperta perché

2 DM, 15.

3 Omeliadel Santo Padre Francesco, Basilica Vaticana, 11 aprile 2015, https://w2.vatican.va/ content/francesco/it/homilies/2015/documents/papa-francesco_20150411_omelia-vespridivina-misericordia.html (accesso il 04.08.2017). 
è una realtà dinamica. Essa appartiene all'essenza stessa di Dio e come tale la mente umana la può solo cogliere e descrivere nei suoi aspetti, ma non circoscriverla in una formula matematica. La misericordia sfuggirà sempre a qualsiasi formula stereotipa, perché indica l'agire stesso di Dio che non potrà mai essere esaurito. La sua ricchezza è infinita, e permane come sorgente inesauribile dell'amore di Dio. Per alcuni versi, potrebbe essere utile applicare alla misericordia quanto un grande santo poeta e dottore della Chiesa, il diacono s. Efrem il Siro scriveva a proposito della Parola di Dio:

Siamo proprio come gli assetati che devono a una fonte. La tua parola offre molti aspetti diversi, come numerose sono le prospettive di coloro che la studiano... Ha nascosto nella sua parola tutti i tesori, perché ciascuno di noi trovi un a ricchezza in ciò che contempla... Colui al quale tocca una di queste ricchezze non creda che non vi sia altro nella parola di Dio oltre ciò che ha trovato. Si renda conto piuttosto che egli non è stato capace di scoprirvi se non una sola cosa fra molte altre. Dopo essersi arricchito nella parola non creda che questa venga da ciò impoverita. Incapace di esaurirne la ricchezza, renda grazie per la sua immensità. Rallegrati perché sei stato saziato, ma non rattristarti per il fatto che la ricchezza della parola ti superi. Colui che ha sete è lieto di bere, ma non si rattrista perché non riesce a prosciugare la fonte. È meglio che la fonte soddisfi la tua sete, piuttosto che la sete esaurisca la fonte. Se la tua sete è spenta senza che la fonte sia inaridita, potrai bervi di nuovo ogni volta che ne avrai bisogno. Se invece saziandoti seccassi la sorgente, la tua vittoria sarebbe la tua sciagura. Ringrazia per quanto hai ricevuto e non mormorare per ciò che resta inutilizzato. Quello che hai preso o portato via è cosa tua, ma quello che resta è ancora tua eredità. Ciò che non hai potuto ricevere subito a causa della tua debolezza, ricedilo in altri momenti con la tua perseveranza. Non avere l'impudenza di voler prendere in un sol colpo ciò che non può essere prelevato se non a più riprese, e non allontanarti da ciò che potresti ricevere solo un po' alla volta ${ }^{4}$.

\section{La liturgia spazio reale della misericordia}

La misericordia si presenta ai noi con molti volti che ne raffigurano la sua bellezza, la sua bontà e la realtà che esprime. Tra i tanti, ho scelto in particolare due volti della misericordia che possiamo cogliere come espressione qualificante della sua multiforme realtà. Il primo volto, lo si contempla nella liturgia. È qui che si coglie la verità profonda della misericordia come essenza stessa della Trinità. Se

4 Efrem, Commenti sul Diatesseron 1, 18-19, in: Sources Chrétiennes 121, Éditions du Cerf, Paris 1966, p. 52-53. 
solo avessimo il tempo di verificare quante volte il termine risuona nella liturgia, vedremmo che esso è onnicomprensivo. Nella maggioranza delle collette, la misericordia risulta come l'invocazione più frequente che viene rivolta dai fedeli al Padre perché la conceda in abbondanza come espressione di vita nuova. Una tra le più conosciute: "O Dio che riveli la tua onnipotenza soprattutto con la misericordia e il perdono..." . Altri testi, comunque, riflettono la stessa intensità; si pensi, solo a livello esemplificativo, alla Colletta nella Domenica di Quaresima destinata fin dall'antichità allo scrutinio dei catecumeni: "Dio misericordioso fonte di ogni bene, tu ci hai proposto a rimedio del peccato il digiuno, la preghiera e le opere di carità fraterna; guarda a noi che riconosciamo la nostra miseria e poiché ci opprime il peso delle nostre colpe, ci sollevi la tua misericordia"6. Come si nota: miseria umana e bontà divina si incontrano per dare voce alla misericordia come espressione di liberazione totale.

Nella liturgia eucaristica, inoltre, dall'inizio alla fine, la misericordia costituisce il riferimento costante per accedere purificati e vivere degnamente la celebrazione dei sacri misteri. "Dio abbia misericordia di noi, perdoni i nostri peccati e ci conduca alla vita eterna", sono le parole iniziali del sacerdote all'inizio dell'atto penitenziale. Alla stessa stregua prega per se stesso il sacerdote nella preghiera eucaristica del Canone Romano: "Anche a noi tuoi ministri peccatori, ma fiduciosi nella tua infinita misericordia"; e ancora: "Di noi tutti abbi misericordia, donaci di aver parte alla vita eterna", sono la conclusione della Seconda Preghiera Eucaristica. "Nella tua misericordia a tutti sei venuto incontro perché coloro che ti cercano ti possano trovare", sono l'inizio della Quarta Preghiera eucaristica. Prima del richiamo al segno di pace, la misericordia ritorna di nuovo: "Con l'aiuto della tua misericordia vivremo sempre liberi dal peccato". Insomma, la misericordia lontano dall'essere un semplice richiamo parenetico è l'asse portante della preghiera liturgica.

Nella liturgia, si comprende il valore performativo che la preghiera assume per ottenere misericordia. Forse, l'espressione che più di ogni altra merita essere ricordata in questo contesto è l'invocazione del Salmista più volte ripresa nella liturgia: "Mostraci, Signore, la tua misericordia e donaci la tua salvezza" (Sl 85, 8). In ogni celebrazione liturgica, questa parola diventa davvero reale ed efficace. Nei sacri misteri che la Chiesa celebra, la Trinità si rivela come il mistero dell'insondabile amore eternamente vissuto, che si china con bontà verso i credenti, inondandoli del dono della grazia imperscrutabile che i sacramenti esprimono. Un breve richiamo a questo Salmo permette di comprendere il grande valore

5 XXVI Domenica del Tempo Ordinario, Questa colletta appare già, nell'VIII secolo, tra i testi eucologici del Sacramentario Gelasiano (1198).

6 III Domenica di Quaresima. 
che la preghiera liturgica gli affida. Non è un caso, infatti, che ritroviamo questo Salmo nel periodo dell'Avvento, dove l'intento è quello di indicare il dono più grande del Padre all'umanità: l'incarnazione del Figlio. In questo Salmo ritornano gli elementi fondamentali che compongono la misericordia: perdono (v. 3), conversione (v. 5), salvezza (v. 8), pace (v. 9), gloria (v. 10), amore (v. 11), verità (v. 11), giustizia (v. 11), bene (v. 13), sono richiamati dal Salmista per esprimere la gioia del dono della misericordia divina che manifesta i tratti del volto di Cristo. Il tema del "ritorno" $(s h u b)$ che più volte è presente nel Salmo, non è solo in riferimento alla deportazione del popolo in esilio, ma indica più spiritualmente la conversione e il ritorno alla casa del Padre, che attende impaziente il ritorno di quanti si sono allontanati (cfr Lc 15, 11-32). E, tuttavia, non solo l'uomo ritorna a Dio; anche a Dio si chiede di ritornare verso il suo popolo. Solo in questo modo la misericordia acquista tutta la sua valenza perché incarna il vero volto di Dio che non si lascia andare all'ira, "ma placa il suo sdegno" (v. 5); se la prima dura un istante, la seconda permane in eterno. Insomma, tutto in questo Salmo parla della tenerezza e dell'amore di Dio che parla al cuore dell'uomo perché riconosca la sua misericordia.

Con ragione, pertanto, Giovanni Paolo II poteva scrivere:

La Chiesa professa la misericordia di Dio, la Chiesa ne vive nella sua ampia esperienza di fede ed anche nel suo insegnamento, contemplando costantemente Cristo, concentrandosi in lui, sulla sua vita e sul suo Vangelo, sulla sua croce e risurrezione, sull'intero suo mistero [...] Gran significato ha in questo ambito la costante meditazione della parola di Dio e, soprattutto, la partecipazione cosciente e matura all'Eucaristia e al sacramento della penitenza o riconciliazione. L'Eucaristia ci avvicina sempre a quell'amore che è più potente della morte [...] Lo stesso rito eucaristico, celebrato in memoria di colui che nella sua missione messianica ci ha rivelato il Padre, per mezzo della parola e della croce, attesta quell'inesauribile amore in virtù del quale egli desidera sempre unirsi ed immedesimarsi con noi, andando incontro a tutti i cuori umani. È il sacramento della penitenza o riconciliazione che appiana la strada ad ognuno, perfino quando è gravato di grandi colpe. In questo sacramento ogni uomo può sperimentare in modo singolare la misericordia, cioè quell'amore che è più potente del peccato ${ }^{7}$.

Nell'azione liturgica, pertanto, il credente si pone nella contemplazione della misericordia di Dio. Nel silenzio dell'ascolto, percepisce la potenza del mistero che gli si fa incontro e viene abilitato per grazia a diventarne segno concreto con la sua testimonianza nel mondo. Lontano dallessere un momento che distoglie

7 DM, 13. 
dal vivere la misericordia, la liturgia ne permette la sua giusta attuazione. Porsi in ginocchio dinanzi alla grandezza dellamore misericordioso della Trinità è il segno tangibile della trasformazione che avviene nel cuore di ogni persona che ha ascoltato l'annuncio del Vangelo della misericordia e vi ha aderito con fede.

Il tempo liturgico, dunque, è a pieno titolo il tempo della misericordia.

\section{La misericordia come testimonianza}

Un secondo volto della misericordia si esprime nella vita dei credenti. Tanti sono i volti della misericordia che si dovrebbero ammirare, quanti sono i volti dei discepoli di Cristo. Certo, il limite del peccato è sempre all'erta. Per ognuno di noi la tentazione di rinchiudersi in se stesso, nell'indifferenza, e nella stanchezza sono sempre allerta; eppure, sappiamo che la misericordia agisce come il "cuore inquieto" di cui parlava sant'Agostino. Essa non lascia tranquillo nessuno fino a quando non si è diventati strumento di misericordia. Le parole di Papa Francesco sono una provocazione permanente per la nostra vita di fede: "Nelle nostre parrocchie, nelle comunità, nelle associazioni e nei movimenti, insomma, dovunque vi sono dei cristiani, chiunque deve poter trovare unoasi di misericordia". Dovrebbe essere proprio così. La presenza attiva del credente richiede di essere permeata dalla misericordia con la quale professa la fede, che lo rende discepolo di Cristo; rende evidente l'amore, che lo spinge ad agire; e proclama la speranza, che gli permette di essere sempre in cammino verso il compimento della promessa. La misericordia traccia un percorso per indicare alla Chiesa dove sentirsi profondamente impegnata. Le opere di misericordia, ad esempio, non sono costrizione, ma testimonianza concreta di come i cristiani sono chiamati ad alleviare la sofferenza dei fratelli. La misericordia pertanto è un atto, un'azione, un segno concreto che viene vissuto.

In questo contesto merita riflettere su come possiamo vivere e rendere sempre più attuali le opere di misericordia corporale e spirituale. È una vera sfida quella di dare voce e forma alla fecondità della misericordia. Essa si esprime nel tentativo non solo di reinterpretare le sette opere di misericordia corporale e spirituale che conosciamo, ma nella capacità di scoprirne e inventarne delle nuove. Per la Chiesa questa strada è un obbligo perché le permette di comprendere se stessa realmente inserita nella storia che vive e nella quale è chiamata ad essere "segno e strumento" della misericordia del Padre. È ovvio che dinanzi alla parabola che ricorda le opere di misericordia corporale, che Gesù stesso ci ha lasciato quasi

8 Misericordiaevultus, $12[\mathrm{MV}]$,https://w2.vatican.va/content/francesco/it/apost_letters/ documents/papa-francesco_bolla_20150411_misericordiae-vultus.html(accesso il 04.08.2017). 
fosse un suo testamento ${ }^{9}$, ogni cristiano è chiamato a viverle alla lettera, sine glossa. Dare da mangiare a chi ha fame e bere a chi ha sete; ospitare chi è senza casa e assistere chi è nella sofferenza della malattia o della mancanza di libertà. Eppure, mi domando: come si può interpretare oggi lopera di misericordia "vestire i nudi"? La Sacra Scrittura non ci lascia molto spazio per aggiornare la nostra interpretazione. È necessario comprendere bene il testo che conosciamo per affidargli il suo senso completo. Subito all'inizio della Genesi troviamo il lamento di Adamo: "Ho udito la tua voce nel giardino: ho avuto paura perché sono nudo e mi sono nascosto" $(\mathrm{Gn} 3,10)$. A questo momento di turbamento a seguito del peccato che rovina la relazione con Dio e quelle interpersonali, viene subito in aiuto la misericordia del Padre; commenta il libro della Genesi: "Il Signore Dio fece all'uomo e a sua moglie tuniche di pelli e li vestì" (Gn 3, 21). Forse, è proprio qui il primo richiamo allazione misericordiosa di Dio che toglie Adamo ed Eva dall'imbarazzo della loro nudità. Non sono loro che si vestono, è Dio che provvede loro il vestito! Il nascondimento provocato dalla nudità che aveva creato isolamento, paura e vergogna, viene superato dall'abito che restituisce una presenza sociale. Ė la stessa cosa che succede con Noè ubriaco, rimane nudo ( $\mathrm{cfr} G \mathrm{Gn} 9,21)$. Saranno i suoi figli Sem e Jafet camminando a ritroso a coprire la nudità del padre perché non sia svergognato dinanzi a nessuno ( $\mathrm{cfr}$ Gn 9, 23). Il senso che si può cogliere è nitido: vestire i nudi significa restituire loro dignità. I Vangeli non hanno timore a parlare di Gesù nudo sulla croce. La sua tunica viene messa a sorte e lui, inchiodato al Calvario, condivide le sorti di chi è senza dignità. Tocca alla Chiesa, che s. Cipriano identifica nella tunica di Cristo, rivestire quanti sono nudi per restituire a tutti la dignità di figli. "Ero nudo e mi avete vestito", pertanto, provoca a individuare le nuove forme di povertà ed emarginazione sociale che impediscono alle persone di avere dignità. Certamente, non avere un lavoro, non avere una casa, non avere un salario rispettoso del lavoro, essere discriminati per la propria fede... sono tutti elementi che impediscono di avere dignità. Come la Chiesa e il cristiano possono contribuire a restituire dignità a quanti sono nudi è un imperativo che non può coglierci di sorpresa, ma che impone vigilanza dinanzi alle nuove forme di povertà.

Alla stessa stregua, dovremmo entrare nel merito delle opere di misericordia spirituale. Come può essere vissuta oggi l’opera che dice: "ammonire i peccatori". In un contesto culturale come il nostro in cui l'individualismo ha fatto perdere di vista la responsabilità per l'altro, come possiamo essere in grado di far compren-

9 Non si dimentichi che questo capitolo 25 di Matteo è l'ultimo discorso che Gesù compie. La parabola raccontata sul giudizio finale può essere interpretata facilmente come l'ultimo insegnamento lasciato da Gesù ai discepoli nella teologia di Matteo; cfr. J. Gnilka, Il Vangelo di Matteo II, Brescia 1991, p. 533-554. 
dere la mancanza di responsabilità sociale che investe ormai tanti ambiti della vita pubblica? Come poter far comprendere a una persona che sbaglia, che seguendo quella strada non compie il bene e tanto meno realizza la sua identità personale? Il relativismo che ci accompagna per cui è bene ciò che ognuno ritiene tale, come può essere superato in vista di un bene comune che trova nella legge scritta nel profondo del cuore di ognuno e nella comune natura, il suo fondamento oggettivo? Insomma, da ogni parte consideriamo le opere di misericordia, siamo comunque spinti a una loro più fattiva interpretazione per renderle attuali e concrete nelle mutate condizioni sociali e culturali in cui ci troviamo.

Potremmo essere tentati di andare subito al concreto e anche qui costruire opere; eppure, l'indicazione che proviene è anzitutto quella di essere consapevoli e coscienti dellesigenza di non abituarci mai alla misericordia. Come la misericordia non si lascia definire, così essa non si lascia circoscrivere in un ospedale, in una scuola, in una mensa o in un ostello. No, la misericordia è piuttosto un cuore inquieto che cerca continuamente il volto di Cristo in quello del fratello e non si arrende fino a quando, per dirla con le parole di Gesù nella parabola del buon samaritano: "ne ebbe compassione. Gli si fece vicino, gli fasciò le ferite, versandovi olio e vino; poi, caricatolo sopra il suo giumento, lo portò a una locanda e si prese cura di lui" (Lc 10, 33-34).

Prendersi cura del fratello: è questo il primo impatto con la misericordia. Per alcuni aspetti, potremmo individuarne alcuni tratti nella vita quotidiana dei primi cristiani. È conosciuto il racconto di Pietro e Giovanni che dopo la Pentecoste si recarono al tempio per la preghiera (At 3, 1-7). Il racconto è ricco di particolari che meritano di essere ricordati: uno storpio fin dalla nascita chiedeva l'elemosina alla porta "Bella" del tempio. Vedendo arrivare Pietro e Giovanni "li pregava per avere unelemosina”. Non dimentichiamo che dietro il gesto abitudinario, si nasconde però la parola chiave: $\dot{\lambda} \lambda \varepsilon \eta \mu o \sigma u ́ v \eta$. In greco, misericordia, si dice elemosina! Siamo chiamati quindi a vedere dietro l'atto del chiedere una moneta, la richiesta di ottenere misericordia. Ed ecco come la misericordia si esprime in Pietro: "Fissando lo sguardo su di lui [...] disse: guarda verso di noi". Quello storpio sperava di ottenere una somma; Pietro, invece, gli offre misericordia. Il suo non fu un ingresso frettoloso nel tempio, e neppure manifestò insofferenza per un altro questuante davanti alla porta che gli chiedeva i soldi. Pietro guarda il mendicante, si accorge di lui e gli rivolge la parola. Non ha né oro né argento, ma quanto possiede, cioè la sua fede e il suo amore per Gesù, lo offre e lo condivide con il povero storpio. L'insegnamento è profondo. La misericordia è anzitutto condivisione che coinvolge la vita stessa della persona. Non in primo luogo una banconota, ma latto della condivisione di ciò che si ha di più importante. La misericordia punta diritto all'essenziale nella vita e a ciò che uno pensa di avere come più prezioso. Infine, Pietro tocca il mendicante: "lo prese per la mano destra e lo sollevò". 
Quante volte abbiamo sentito papa Francesco parlare dell'esigenza di "toccare la carne di Cristo". La misericordia è un prendere per la mano e sollevare. Ė quindi un impegno concreto che aiuta a rialzarsi dalla condizione di povertà per recuperare la dignità perduta. Passare accanto a una persona, accorgersi della sua necessità, iniziare un colloquio, guardarla negli occhi, toccarla, condividere, e aiutare a rialzarsi... ecco tutti i tratti che permettono di mettere in pratica la misericordia. La misericordia, dunque, è la vita quotidiana di ogni credente che desidera essere discepolo del Signore. Una vita che si esprime con l'attenzione, la vicinanza, la solidarietà, la condivisione, la consolazione e il perdono. Ecco perché questo è il tempo della misericordia.

\section{Per concludere}

Il Giubileo ha un carattere straordinario, ma ritengo che abbia inciso in maniera molto forte sulla vita della Chiesa. Lo sguardo, dopo il 20 novembre dovrà essere capace di guardare ancora alla misericordia come il luogo privilegiato dove poter fare esperienza della fede che si ravviva, della speranza che si rafforza, e della carità che non si stanca. Certamente, dopo un Anno Santo così intenso, vissuto nellesperienza diretta di tutte le Chiese nel mondo, la sfida diventa grande. Essa consiste nel verificare come continuare ad essere testimoni di misericordia e come fare della misericordia il cuore dellazione pastorale. Con ragione il magistero recente della Chiesa ha insistito perché la misericordia ritorni ad essere "l'architrave che sorregge la vita della Chiesa" e la sua intera "azione pastorale". Si possono riprendere le parole della bolla Misericordiae vultus che sono un vero spiraglio aperto per l'impegno della pastorale dopo il Giubileo:

Nulla del suo annuncio e della sua testimonianza verso il mondo può essere privo di misericordia. La credibilità della Chiesa passa attraverso la strada dell'amore misericordioso e compassionevole. La Chiesa "vive un desiderio inesauribile di offrire misericordia" [...] Ė giunto di nuovo per la Chiesa il tempo di farsi carico dellannuncio gioioso del perdono. Ė il tempo del ritorno allessenziale per farci carico delle debolezze e delle difficoltà dei nostri fratelli. Il perdono è una forza che risuscita a vita nuova e infonde il coraggio per guardare al futuro con speranza ${ }^{10}$.

Alla Chiesa è stato affidato il "ministero della riconciliazione" (2 Cor 5, 18). È attraverso questo annuncio fattivo che il mondo può credere all'amore di Dio che ha donato se stesso per la salvezza dell'uomo. Non potrà mai mancare, pertanto, 
la "parola di riconciliazione" $(2$ Cor 5,19$)$ con la quale la Chiesa è impegnata a far rispendere nel mondo la luce della misericordia offerta con la presenza di Cristo Redentore. Il tempo della misericordia, dunque, non si conclude con la fine del Giubileo, ma si staglia dinanzi a noi come impegno per il futuro della Chiesa nel mondo. Una responsabilità che non può essere domandata ad altri, perché impegna in prima persona ogni cristiano. Il cuore di ognuno, pertanto, si deve riempire di gioia per il grande dono che ci è stato fatto di portare a tutti il Vangelo della misericordia.

\section{Abstract}

\section{This is the Time of Mercy}

Mercy cannot be enclosed in a unique, linguistic term, which exhausts fully its meaning. It is an open word because it expresses a very dynamic reality.

Mercy belongs to the essence of God. The human being can grasp Mercy in its aspects. Mercy presents to us in different faces, revealing the God's beauty, goodness and reality. In particular, we concentrate on two faces of Mercy. The first one we contemplate in the Liturgy of the Church. The second is present in life of believers: for Mercy has as many faces, as many disciples has Jesus Christ.

The Time of Mercy does not end with the closure of the Jubilee of Mercy, but becomes to us a challenge for the future. We need to rethink our tasks and to decide, how to continue our testimony of being merciful. We should ask ourselves: how to make of this testimony the heart of our pastoral work. 